\title{
Oxidative Stress Status in Testis of Type-2 Diabetic Rats Treated with Delta-9-Tetrahydrocannabinol
}

\author{
Ebrar Tutar' ${ }^{\mathbb{D}}$, Beti Pesen' ${ }^{(\mathbb{D})}$, Zeynep Mine Coskun Yazici1",* (D), Sema Bolkent ${ }^{2}$ (D) \\ 'Demiroglu Bilim University, Faculty of Arts and Sciences, Department of Molecular Biology and Genetics, Istanbul, Turkey \\ ${ }^{2}$ Istanbul University-Cerrahpasa, Faculty of Cerrahpasa Medicine, Department of Medical Biology, Istanbul, Turkey
}

ORCID IDs of the authors: E.T. 0000-0003-2273-7487; B.P. 0000-0003-1449-9749; Z.M.C.Y. 0000-0003-4791-6537; S.B. 0000-0001-8463-5561

Please cite this article as: Tutar E, Pesen B, Coskun Yazici ZM, Bolkent S. Oxidative Stress Status in Testis of Type-2 Diabetic Rats Treated with Delta-9-Tetrahydrocannabinol. Eur J Biol 2021; 80(2): 91-96. DOI: 10.26650/EurJBiol.2021.1001540

\begin{abstract}
Objective: Type-2 diabetes (T2D) is a multifactorial disease that occurs as a result of impaired insulin secretion and increased glucose. Researchers emphasize that oxidative stress prevention and improvement strategies are important in the general treatment of T2D. It is reported that delta-9-tetrahydrocannabinol (THC), a component of the cannabis plant, has positive effects against oxidative stress and inflammation. Therefore, this study explored the inhibitory effects of THC on oxidative damage of testis in type-2 diabetic rats.

Materials and Methods: Adult Spraque-Dawley rats were separated into 4 groups. In the control group, physiological saline was given intraperitoneally. In the T2D model group (T2DM), streptozotocin (STZ) + nicotinamide (NAD) was administered intraperitoneally. Three $\mathrm{mg} / \mathrm{kg} /$ day THC was given to the THC and T2DM+THC groups for 7 days, intraperitoneally. Glutathione $(\mathrm{GSH})$, malondialdehyde (MDA), and protein carbonyl levels (PCO), and superoxide dismutase (SOD) activity were measured spectrophotometrically in testicular tissue. Total oxidant and antioxidant status were determined by ELISA.

Results: Testicular GSH level and SOD activity were significantly decreased in T2D, while MDA and PCO levels increased. In contrast to this effect, the THC treatment increased GSH levels and SOD activity in diabetics, but decreased MDA and PCO levels.

Conclusion: According to the study results, THC may have an oxidative stress inhibitory effect on diabetic rat testis.

Keywords: Diabetes, tetrahydrocannabinol, testis, oxidative stress
\end{abstract}

\section{INTRODUCTION}

Type-2 diabetes (T2D), the most common type, is a chronic disease characterized by high blood sugar. Steroidogenic and spermatogenic dysfunctions are complications of T2D in men. Some researchers suggest that the use of anti-diabetic drugs has a restorative effect on them $(1,2)$. Tian et al. suggested that reactive oxygen species and autophagy act a part in the pathophysiology of diabetes-induced male infertility (3). Consistent with the studies by Tian et al. (3), Ranjan et al. showed that T2D causes testicular dysfunction in mice (4).
The cannabis plant has been used for thousands of years as a medicinal agent and contains approximately 540 natural compounds. One of the most potent bioactive components of cannabis is delta-9-tetrahydrocannabinol (THC) (5). Vella et al. reported that THC could lead to the prevention of cardiovascular dysfunction by reducing blood glucose concentrations and oxidative stress in streptozotocin (STZ)-diabetic Wistar-Kyoto rats given THC (6). In the previous study, THC ameliorated hyperlipidemia and hyperglycemia in type-2 diabetic rats (7). In a study administered THC to multiple lowdose STZ-induced autoimmune diabetic animals, researchers suggested that multiple low dose STZ could 
cause beta-cell damage, but the administration of THC had the ability to reduce the severity of the autoimmune response (8). Cantele et al. (9) suggested that Cannabis sativa L. may be used as a source of natural antioxidant. In addition, an in vitro study by Raja et al. (10), showed that THC has a high potential to combat oxidative stress, while no appreciable antioxidant activity of cannabidiol (CBD) was detected. Thus, researchers reported that cannabis extracts exhibited significant antioxidant activity depending on their THC and CBD ratios. Unfortunately, there is no information about the effect of THC on type-2 diabetic rat testis. Therefore, this study examined the effect of THC on oxidative damage in the testis of rats with T2D.

\section{MATERIALS AND METHODS}

\section{Experimental Procedures}

Experiments were performed on 8-10 week old male Sprague-Dawley rats ( $n=24$, average weight 214 g). All experimental procedures were performed using approved methods according to the standards of the Istanbul University Animal Research Local Ethics Committee (approval number: 103). All animals were fed and drank water ad libitum and were housed at standard status ( $12 \mathrm{~h}$ light-dark cycle). Twenty-four male rats were randomly selected and assigned to one of the four groups: control, T2D model (T2DM), THC, and T2DM+THC. Saline was intraperitoneally injected into the control group. A single dose of $85 \mathrm{mg} / \mathrm{kg}$ nicotinamide (NAD; Sigma-Aldrich) and 15 minutes later $65 \mathrm{mg} / \mathrm{kg} \mathrm{STZ} \mathrm{(Sigma-Aldrich)} \mathrm{were} \mathrm{injected} \mathrm{intraperi-}$ toneally into the T2DM group (11). Blood glucose levels were determined $72 \mathrm{~h}$ after STZ+NAD injections, and animals with a blood glucose concentration $\geq 200 \mathrm{mg} / \mathrm{dL}$ were considered type 2 diabetic.

THC (3 mg/kg/day; Lipomed) was administered to the THC group and the T2DM+THC group for 7 days, intraperitoneally. Testicular tissue samples were taken from rats under anesthesia 15 days after THC injection. The tissue samples were fixed with nitrogen $\left(-196^{\circ} \mathrm{C}\right)$, and then kept at $-86^{\circ} \mathrm{C}$.

\section{Biochemical Analysis}

Tissue homogenates $(10 \% \mathrm{w} / \mathrm{v})$ were obtained from frozen testicular tissue samples in ice-cold saline. The specimens were centrifuged at $5.000 \times \mathrm{g}$ for ten minutes, and supernatants were obtained and used directly to measure biochemical parameters. Glutathione (GSH), malondialdehyde (MDA), and protein carbonyl levels (PCO), and superoxide dismutase (SOD) activity were assessed using the Beutler, Buege and Aust, Reznick and Packer, and Sun et al. methods, respectively (12-15).

The GSH level was detected by following the rate of decline of 5.5'-dithiobis-2-nitrobenzoic acid (DTNB). Shortly, precipitating solution ( $\mathrm{Na}_{2}$ EDTA, glacial metaphosphoric acid, $\mathrm{NaCl}$ ) were added to testis tissue samples and mixed. The reaction mixture was incubated. And then samples were centrifuged. Phosphate and DTNB solutions were added to the supernatant. The difference in absorbance was read at $412 \mathrm{~nm}$. The GSH concentration in samples was calculated from a standard curve. The GSH concentration in testis tissue was expressed in $\mathrm{nmol} / \mathrm{mg}$ of protein.

MDA levels were analyzed by a thiobarbituric acid reacting substances assay. Two-hundered fifty $\mu \mathrm{L}$ of testis tissue sample was added and then mixed with $1 \mathrm{~mL}$ of $30 \%$ trichloroacetic acid, $0.2 \mathrm{~mL}$ of $5 \mathrm{M} \mathrm{HCl}$, and $1.5 \mathrm{~mL}$ of $0.75 \%$ thiobarbituric acid. The specimens were incubated at $100^{\circ} \mathrm{C}$ for 20 minutes. Following the incubation period, the specimens were centrifuged for 10 min, and samples read at $532 \mathrm{~nm}$, spectrophotometrically.

PCO reacts with 2, 4-dinitrophenyl-hydrazine (DNPH) to generate chromophoric dinitrophenylhydrazones (1:4, tissue: DNPH). DNPH was dissolved in hydrochloric acid. After the DNPH reaction, proteins were precipitated with an equal volume of $20 \%$ $(w / v)$ trichloroacetic acid. Then, obtained pellets were washed with an ethanol/ethyl acetate mixture. Lastly, the PCO precipitates were dissolved in guanidine- $\mathrm{HCl}$ solution. The absorbance values were recorded at $360 \mathrm{~nm}$. The PCO concentration was counted using the molar extinction coefficient of DNPH $(\varepsilon=$ $\left.22.000 \mathrm{M}^{-1} \mathrm{~cm}^{-1}\right)$.

The method of SOD enzyme activity involves the inhibition of nitroblue tetrazolium (Sigma-Aldrich) reduction with xanthine/ xanthine oxidase (Sigma-Aldrich). The SOD activity was detected by colorimetric evaluation of soluble formazan dye formation at $560 \mathrm{~nm}$. One unit of SOD is described as the amount causing 50\% inhibition in the nitroblue tetrazolium reduction rate. The SOD enzyme activity was presented as $\mathrm{U} / \mathrm{mg}$ protein in the testis.

Total protein levels were detected using the Bradford assay method in which $40 \mu \mathrm{L}$ of testis tissue specimens was added to $2 \mathrm{~mL}$ of Bradford reagent. At the end of the incubation period, the absorbance was read at $595 \mathrm{~nm}$ (16). A bovine serum albumin standard was used for calculation, and results were presented as $\mathrm{mg}$ protein $/ \mathrm{mL}$.

\section{Analysis of Total Antioxidant and Oxidant Statutes}

Total antioxidant status and total oxidant status were detected using commercial kits (Rel Assay). Measurements were made according to the kit procedure. The oxidative stress index was checked out according to the following formula.

Oxidative stress index $=\frac{\text { Total oxidant status }\left(\mathrm{mmol} \mathrm{H}_{2} \mathrm{O}_{2} \mathrm{Eq} / \mathrm{L}\right)}{\text { Total antioxidant status }(\mathrm{mmol} \mathrm{Trolox} \mathrm{Eq} / \mathrm{L})} \times 100$

\section{Statistics}

Data was declared as the mean \pm standard error of the mean (SEM). The non-parametric Kruskal-Wallis and Mann-Whitney tests were benefited because the data failed to passa normality test. The differences were considered as significant at a $P$-value $<0.05$ (Prism 5, GraphPad Software).

\section{RESULTS}

In this study, there were significant differences in GSH, MDA, and PCO levels, and SOD activity among all groups $(p<0.05$, $p<0.01, p<0.01$ and $p<0.05$, respectively) (Figures $1-4)$. The 


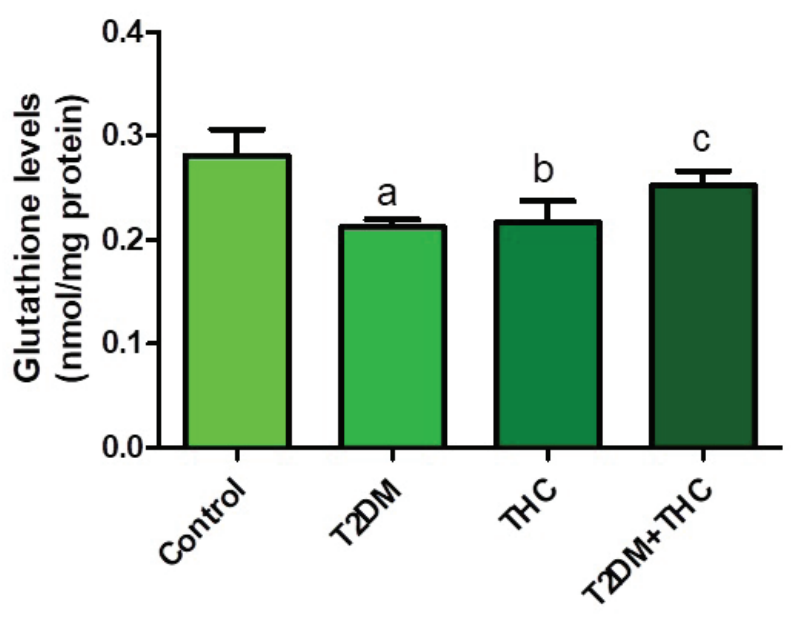

Figure 1. Changes in glutathione levels in testis tissue. T2DM, type-2 diabetes model; THC, delta-9-tetrahydrocannabinol. Values are shown as means \pm SEM. ${ }^{a} p<0.01$ vs Control group, ${ }^{b} p<0.05$ vs Control group, ${ }^{c} p<0.01$ vs T2DM group.

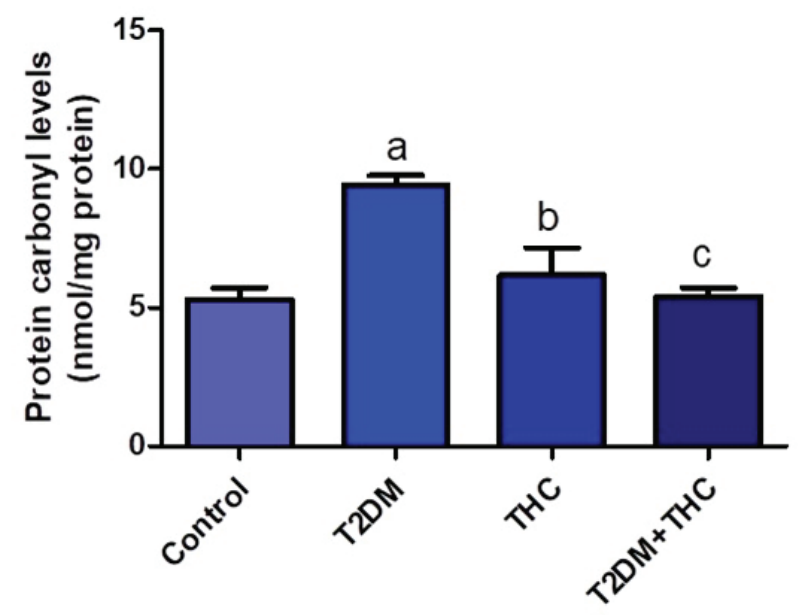

Figure 3. Protein carbonyl levels in testis tissue of rats. T2DM, type-2 diabetes model; THC, delta-9-tetrahydrocannabinol. Values are shown as means \pm SEM. ${ }^{a} p<0.01$ vs Control group, ${ }^{\mathrm{b}} p<0.05$ vs T2DM group, ${ }^{\mathrm{c}} p<0.01$ vs T2DM group.

GSH level in the testis tissue decreased in the T2DM group as compared to the control rats $(p<0.01)$. THC administration significantly increased testis GSH level in the T2DM+THC group as compared to the T2DM group ( $p<0.01)$. MDA, a lipid peroxidation marker, increased in T2DM rats when compared with control animals $(p<0.01)$. However, the increase in testis MDA level of the T2DM group decreased significantly with THC treatment $(p<0.01)$. Interestingly, when compared to control and THC groups, the lowest MDA level was detected in the T2DM+THC group ( $p<0.05$ and $p<0.01$, respectively). Similar to MDA level, type-2 diabetes significantly raised the PCO level when com-

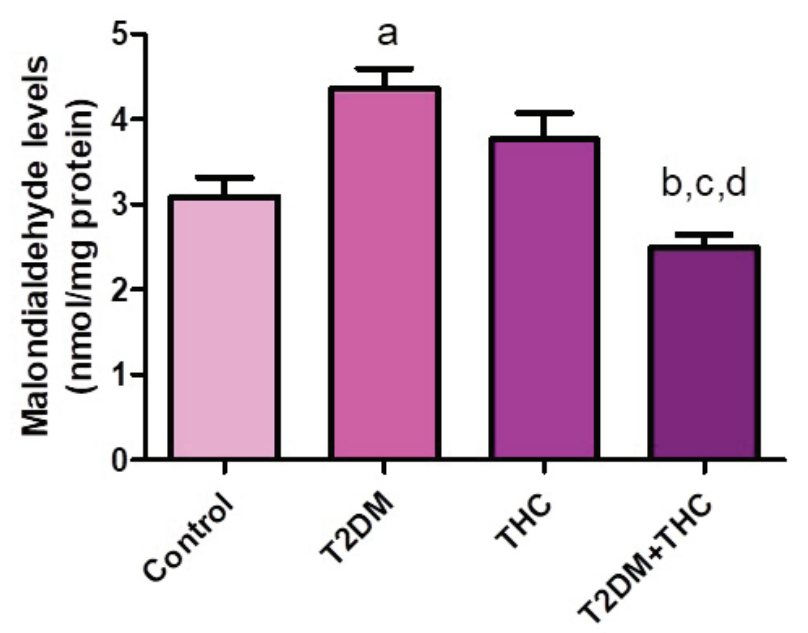

Figure 2. Testicular malondialdehyde levels. T2DM, type-2 diabetes model; THC, delta-9-tetrahydrocannabinol. Values are shown as means \pm SEM. ${ }^{a} p<0.01$ vs Control group, ${ }^{b} p<0.05$ vs Control group, ${ }^{c} p<0.01$ vs T2DM group, ${ }^{\mathrm{d}} p<0.01$ vs THC group.

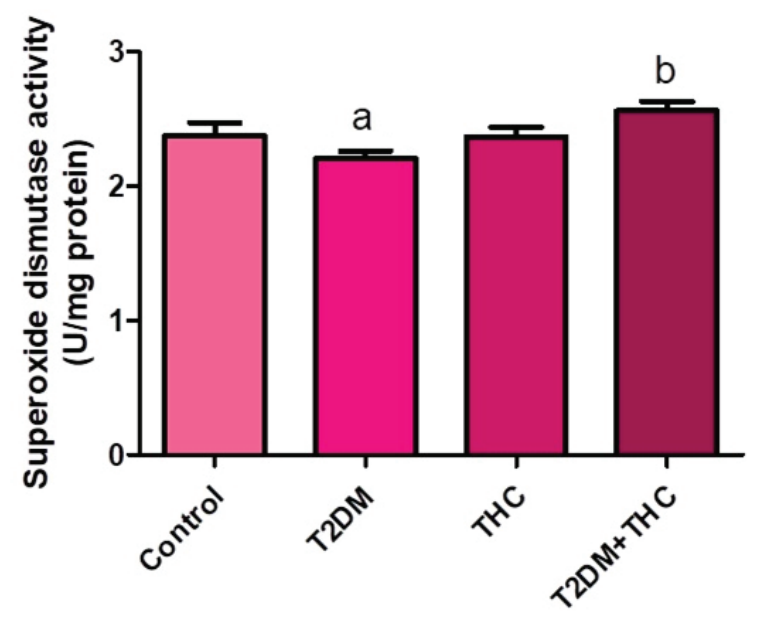

Figure 4. Measurement of superoxide dismutase enzyme activities in testis tissue. T2DM, type-2 diabetes model; THC, delta-9-tetrahydrocannabinol. Values are shown as means \pm SEM ${ }^{\mathrm{a}} p<0.05$ vs Control group, ${ }^{\mathrm{b}} p<0.01$ vs T2DM group.

pared to control rats $(p<0.01)$. Also, the testis PCO levels showed a significant decrease in the T2DM+THC group as compared to T2DM rats $(p<0.01)$. Testis SOD activity decreased in T2DM animals when compared with the control group $(p<0.05)$. However, THC treatment significantly increased SOD activity in the T2D$M+$ THC group when compared with the T2DM group $(p<0.01)$.

Compared to the control animals, the total antioxidant status value showed an insignificant decrease in the diabetes group, while the total oxidant status value showed an insignificant increase. However, when the THC treatment was applied to the type-2 diabetes group it brought the total antioxidant and ox- 
Table 1. Measurements of total antioxidant and oxidant statutes, and oxidative stress index in testis.

\begin{tabular}{|c|c|c|c|}
\hline & $\begin{array}{l}\text { Total Antioxidant Status* } \\
\text { (mmol Trolox Equiv. /L) }\end{array}$ & $\begin{array}{l}\text { Total Oxidant Status* } \\
\text { ( } \mu \mathrm{mol} \mathrm{H}_{2} \mathrm{O}_{2} \text { Equiv./ L) }\end{array}$ & Oxidative Stress Index \\
\hline Control & $0.40 \pm 0.10$ & $14.68 \pm 4.18$ & $26388 \pm 12090$ \\
\hline T2DM & $0.11 \pm 0.04$ & $28.46 \pm 4.52$ & $57909 \pm 21913$ \\
\hline THC & $0.20 \pm 0.07$ & $14.69 \pm 4.49$ & $16304 \pm 5713$ \\
\hline $\mathrm{T} 2 \mathrm{DM}+\mathrm{THC}$ & $0.45 \pm 0.12$ & $14.23 \pm 5.20$ & $15777 \pm 9635$ \\
\hline$p$ value & NS & NS & NS \\
\hline
\end{tabular}

idant status values closer to the control group. Similar to the total oxidant status results, the oxidative stress index value was insignificantly increased in T2DM groups as compared with the control group. In addition, the oxidative stress index value was lower in the T2DM+THC group than in the T2DM group (Table 1).

\section{DISCUSSION}

This study examined the effects of THC on STZ+NAD-induced diabetic rat testis. The STZ+NAD model of type 2 diabetes was used to create and exhibit symptoms similar to human non-insulin-dependent diabetes mellitus (NIDDM) (11). Numerous studies have shown that the diabetic rat model is useful in studies of different aspects of diabetes $(17,18)$.

Oxidative stress has been recognized as a serious marker for the pathogenesis, and development of type-2 diabetes (19). It has been suggested that hyperglycemia may contribute to increased reactive oxygen species formation and that increased oxidative stress may cause impaired insulin secretion. It has also been emphasized that the inhibition of excessive reactive oxygen species production is very important for delaying the onset of diabetes (20). Studies showed that diabetes causes oxidative damage in rat testis. The oxidative damage in diabetic animals was confirmed by elevations in MDA level and diminishment in GSH level (21-23). Thyagaraju and Muralidhara suggested that PCO levels were higher in diabetic testis and liver tissues than non-diabetics (23). However, testicular SOD activity showed a decrease in diabetic rats (24). A part of a nonenzymatic antioxidant defense system, GSH, protects macromolecules from oxidative damage. In a study by Abdullah et al., it was reported that GSH improves diabetes-induced degraded testicular morphology and sperm quality, thanks to its antioxidant properties (25). Choubey et al. reported that adiponectin reduced oxidative stress and had a protective role against testicular dysfunction in their study in high-fat diet+STZ-induced type-2 diabetic mice (26). In the same research, it stated that the treatment applied to mice decreased the increased lipid peroxidation caused by diabetes and elevated SOD activity. In the current study, T2D decreased GSH levels in testis tissue. MDA, a lipid peroxidation marker, showed an increase in T2DM rats, unlike GSH levels.
Similar to MDA level, T2D raised the PCO level in testis tissue of rats. The testis SOD activity decreased in T2DM animals similar to GSH level.

There are other studies which report that THC has antioxidant effects on various tissues $(27,28)$. Also, Vella et al. suggested that the administration of THC may lead to improvements in cardiovascular dysfunction by providing antihyperglycemic and antioxidant effects in diabetic animals (6). It also showed that THC decreased serum MDA levels in diabetic animals. It has been suggested that consumption of white tea prevents testicular oxidative damage by reducing carbonyl groups and lipid peroxidation and increasing the total GSH level in prediabetic rats, thus it was emphasized that sperm quality can be restored (29). Similar to the aforementioned studies, THC administration increased testis GSH level and SOD activity in the diabetic group. The increase in testis MDA and PCO levels of the T2DM group decreased with THC treatment. On the contrary, Mandal and Das (30) reported in their study on healthy mice that THC damaged testicular tissues. It has even been shown that this detrimental effect is more pronounced at low doses than at higher doses. Further, the researchers observed a healing effect and repair in mouse testicles treated with THC treatment at high doses and withdrawal of drug treatments. They suggested that this might be due to the antioxidant defense system. Similarly, this study observed the effects of THC on testis 15 days after THC treatment was terminated. It showed that THC treatment administered to healthy individuals was not as effective as that administered to diabetic rats. It could be because the extra synthesis of the endogenous antioxidant defense system as a protective defense function of $\mathrm{THC}$ in reducing the high oxidative stress caused by diabetes.

Researchers investigated the antioxidant mechanisms of THC and $\mathrm{CBD}$ and reported that both molecules have antioxidant properties. However, they emphasized that the antioxidant potential of THC is higher than that of CBD (31). While the total antioxidant status value showed a decrease in diabetic rats, the total oxidant status value increased. Although the values were not significant, THC treatment reversed this situation. This may be because THC acts differently in healthy and diabetic individ- 
uals. Thus, the total antioxidant and oxidant status, and oxidative stress index values could only approach the values of the control group. THC may be used as supportive therapy for T2D and its symptoms, especially due to its antioxidative features.

\section{CONCLUSION}

As mentioned above, T2D leads to an increase in oxidative stress markers in testicular tissue. However, T2D causes a reduction in the molecules involved in an antioxidant defense system. This study concludes that the oxidative stress and antioxidant defense markers in testicular tissue move towards healthier levels by administering THC to rats with T2D.

Ethics Committee Approval: This study was approved by the Istanbul University Animal Research Local Ethics Committee (approval number: 103).

Informed Consent: Written consent was obtained from the participants.

Peer Review: Externally peer-reviewed.

Author Contributions: Conception/Design of Study- Z.M.C.Y., S.B.; Data Acquisition- E.T., B.P., Z.M.C.Y.; Data Analysis/Interpretation- Z.M.C.Y., S.B.; Drafting Manuscript- Z.M.C.Y., S.B.; Critical Revision of Manuscript- Z.M.C.Y., S.B.; Final Approval and ACcountability- Z.M.C.Y., S.B., E.T., B.P.

Conflict of Interest: Authors declared no conflict of interest.

Financial Disclosure: Authors declared no financial support.

\section{REFERENCES}

1. Derkach KV, Bakhtyukov AA, Bayunova LV, Zorina II, Shpakov AO. Normalization of testicular steroidogenesis and spermatogenesis in male rats with type 2 diabetes mellitus under the conditions of metformin therapy. Dokl Biol Sci 2020;493(1):110-3.

2. Laakso M. Biomarkers for type 2 diabetes. Mol Metab 2019;27(I):13946.

3. Tian Y, Song W, Xu D, Chen X, Li X, Zhao Y. Autophagy induced by ROS aggravates testis oxidative damage in diabetes via breaking the feedforward loop linking p62 and Nrf2. Oxid Med Cell Longev 2020; 7156579.

4. Ranjan A, Choubey M, Yada T, Krishna A. Nesfatin-1 ameliorates type-2 diabetes-associated reproductive dysfunction in male mice. J Endocrinol Invest 2020;43(4):515-28.

5. Amin MR, Ali DW. Pharmacology of medical cannabis. Adv Exp Med Biol 2019;1162:151-65.

6. Vella RK, Jackson DJ, Fenning AS. $\Delta^{9}$-Tetrahydrocannabinol prevents cardiovascular dysfunction in STZ-diabetic Wistar-Kyoto rats. Biomed Res Int 2017; 7974149.

7. Coskun ZM, Bolkent S. Biochemical and immunohistochemical changes in delta-9-tetrahydrocannabinol-treated type 2 diabetic rats. Acta Histochem 2014;116(1):112-6.

8. Li X, Kaminski NE, Fischer LJ. Examination of the immunosuppressive effect of delta9-tetrahydrocannabinol in streptozotocin-induced autoimmune diabetes. Int Immunopharmacol 2001;1(4):699-712.
9. Cantele C, Bertolino M, Bakro F, Giordano M, Jędryczka M, Cardenia V. Antioxidant effects of hemp (Cannabis sativa L.) inflorescence extract in stripped linseed oil. Antioxidants (Basel) 2020;9(11):1131.

10. Raja A, Ahmadi S, de Costa F, Li N, Kerman K. Attenuation of oxidative stress by cannabinoids and cannabis extracts in differentiated neuronal cells. Pharmaceuticals (Basel) 2020;13(11):328.

11. Masiello P, Broca C, Gross R, Roye M, Manteghetti M, Hillaire-Buys $D$, et al. Experimental NIDDM: development of a new model in adult rats administered streptozotocin and nicotinamide. Diabetes 1998;47(2):224-9.

12. Beutler E. Glutathione: red cell metabolism. A manual of biochemical methods. Grune and Stratton, New York, 1975:112-4.

13. Buege JA, Aust SD. Microsomal lipid peroxidation. Methods Enzymol 1978; 52:302-10.

14. Reznick AZ, Packer L. Oxidative damage to proteins: spectrophotometric method for carbonyl assay. Methods Enzymol 1994;233:35763.

15. Sun Y, Oberley LW, Li Y. A simple method for clinical assay of superoxide dismutase. Clin Chem 1988;34:497-500.

16. Bradford MM. A rapid and sensitive method for the quantitation of microgram quantities of protein utilizing the principle of protein-dye binding. Anal Biochem 1976;72:248-54.

17. Macho-González A, Garcimartín A, López-Oliva ME, Ruiz-Roso, B, Martín de la Torre, I, Bastida, S, et al. Can carob-fruit-extract-enriched meat improve the lipoprotein profile, VLDL-oxidation, and LDL receptor levels induced by an atherogenic diet in STZ-NAD-diabetic rats? Nutrients 2019;11(2):332.

18. Rezagholizadeh L, Pourfarjam Y, Nowrouzi A, Nakhjavani M, Meysamie A, Ziamajidi, N, et al. Effect of Cichorium intybus L. on the expression of hepatic NF-KB and IKK $\beta$ and serum TNF-A in STZ- and STZ+Niacinamide-induced diabetes in rat. Diabetol Metab Syndr 2016; 8:11.

19. Rehman K, Akash MSH. Mechanism of generation of oxidative stress and pathophysiology of type 2 diabetes mellitus: How are they interlinked? J Cell Biochem 2017;118(11):3577-85.

20. Luc K, Schramm-Luc A, Guzik TJ, Mikolajczyk TP. Oxidative stress and inflammatory markers in prediabetes and diabetes. J Physiol Pharmacol 2019;70(6):809-24.

21. Oyenihi AB, Opperman M, Alabi TD, Mpahleni B, Masola B. Centella asiatica alleviates diabetes-induced changes in fatty acid profile and oxidative damage in rat testis. Andrologia 2020;52(10):e13751.

22. Roy VK, Chenkual L, Gurusubramanian G. Protection of testis through antioxidant action of mallotus roxburghianus in alloxan-induced diabetic rat model. J Ethnopharmacol 2015;176:268-80.

23. Thyagaraju BM, Muralidhara. Ferulic acid supplements abrogate oxidative impairments in liver and testis in the streptozotocin-diabetic rat. Zoolog Sci 2008;25(8):854-60.

24. Al-Megrin WA, El-Khadragy MF, Hussein MH, Mahgoub S, Abdel-Mohsen DM, Taha H, et al. Green Coffea arabica extract ameliorates testicular injury in high-fat diet/streptozotocin-induced diabetes in rats. J Diabetes Res 2020;6762709.

25. Abdullah F, Khan Nor-Ashikin MN, Agarwal R, Kamsani YS, Abd Malek M, Bakar NS, et al. Glutathione (GSH) improves sperm quality and testicular morphology in streptozotocin-induced diabetic mice. Asian J Androl 20121;23(3):281-7.

26. Choubey M, Ranjan A, Bora PS, Krishna A. Protective role of adiponectin against testicular impairment in high-fat diet/streptozotocin-induced type 2 diabetic mice. Biochimie 2020;168:41-52.

27. Ismail M, Hasan H, El-Orfali Y, Ismail H, Khawaja G. Anti-inflammatory, antioxidative, and hepatoprotective effects of trans $\Delta 9$-tetrahydrocannabinol/sesame oil on adjuvant-induced arthritis in rats. Evid Based Complement Alternat Med 2018;9365464. 
28. Vacek J, Vostalova J, Papouskova B, Skarupova D, Kos M, Kabelac M, et al. Antioxidant function of phytocannabinoids: molecular basis of their stability and cytoprotective properties under UV-irradiation. Free Radic Biol Med 2021;164:258-70.

29. Oliveira PF, Tomás GD, Dias TR, Martins AD, Rato L, et al. White tea consumption restores sperm quality in prediabetic rats preventing testicular oxidative damage. Reprod Biomed Online 2015;31(4):544-56.
30. Mandal TK, Das NS. Effect of delta-9-tetrahydrocannabinol on altered antioxidative enzyme defense mechanisms and lipid peroxidation in mice testes. Eur J Pharmacol 2009;607(1-3):178-87.

31. Borges RS, Batista JJr, Viana RB, Baetas AC, Orestes E, Andrade MA, et al. Understanding the molecular aspects of tetrahydrocannabinol and cannabidiol as antioxidants. Molecules 2013;18(10):1266374. 